\title{
Impact of Impulse Shot Peening Parameters on Properties of Stainless Steel Surface
}

\author{
M. Wiertel ${ }^{a}$, K. ZAleski $^{b}$, M. Gorgol $^{a}$, A. Skoczylas ${ }^{b}$ And R. Zaleski ${ }^{a, *}$ \\ ${ }^{a}$ Maria Curie-Skłodowska University, Institute of Physics, Department of Nuclear Methods, \\ pl. M. Curie-Skłodowskiej 1, 20-031 Lublin, Poland \\ ${ }^{b}$ Faculty of Mechanical Engineering, Lublin University of Technology, \\ Nadbystrzycka 36, 20-618 Lublin, Poland
}

\begin{abstract}
Shot peening was applied to austenitic stainless steel 1.4541 (EN). The surface treatment was performed at various impact energies $E$, impact densities $j$ and ball diameters $D$. This resulted in improved microhardness, which increases monotonically with the increase of $E, j$ and $1 / D$. However, its changes with $E$ and $j$ achieve saturation at about $400 \mathrm{HV} 0.1$. On the contrary, no saturation is observed in the investigated range for $1 / D$. In the unshot peened 1.4541 (EN) steel, the lifetime component of low intensity was found with use of positron annihilation lifetime spectroscopy (PALS). It corresponds to positron annihilation from delocalized state of positrons in bulk. In the shot peened samples the bulk component is no longer observed. Instead, two types of defects can be identified: vacancy-like defects coupled with edge dislocations and vacancies or their small clusters (consisting $3 \div 5$ vacancies). The results of PALS and hardness testing do not correspond very well, especially in the case of the samples shot peened with balls of varying diameters. The most probable reason for this are different depth profiles of both methods. It seems that the defects, which are responsible for the increase of static microhardness above 400 HV0.1 are located mostly below the surface layer penetrated by positrons.
\end{abstract}

DOI: 10.12693/APhysPolA.132.1611

PACS/topics: 81.65.-b, 81.20.Wk, 62.20.Qp, 61.72.--y, 78.70.Bj

\section{Introduction}

Shot peening (SP) is one of the finishing methods of machine elements. Changes in the surface layer caused by SP result in improved performance of these components, mainly of the fatigue strength. Elements exposed to variable loads are produced, inter alia, of stainless steels. They are mainly used in corrosive environments, especially in the chemical, food, ship, aviation, and chemical industries. Several studies have shown the beneficial effect of SP on the properties of the surface layer of various stainless steels. The $316 \mathrm{~L}$ stainless steel was shot peened by balls having a diameter of $1 \div 2 \mathrm{~mm}$ for $5 \div 25 \mathrm{~min}$ by Azar et al. [1]. It was found that the fatigue resistance increases with time of the treatment and surface hardness changes from the initial value of $350 \mathrm{HV}$ to $480 \mathrm{HV}$ after SP. The surface roughness increases with time of SP for approximately $10 \mathrm{~min}$ but further treatment results in its reduction. The resistance to pitting corrosion also improves as a result of SP. The impact of SP on the residual stress and microstructure was found by Feng et al. [2] in the S3220S stainless steel. SP was carried out in two stages there: first using cast steel balls and then - ceramic balls. The corrosion resistance of joints of $904 \mathrm{~L}$ austenitic stainless steel made by laser welding and shot peened with balls of $0.8 \mathrm{~mm}$ in diameter for 6 min was studied by Nasiłowska et al. [3]. The treatment resulted in the increase of microhardness of the

\footnotetext{
*corresponding author; e-mail: radek@zaleski.umcs.pl
}

surface layer and corrosion resistance by about $100 \%$ and $75 \%$, respectively. Summarizing, the modification of the surface layer caused by SP favorably affects the fatigue strength and corrosion resistance of stainless steels.

One of the nondestructive methods, which allows to connect the properties of the materials with changes of the microstructure of the surface layer, is positron annihilation lifetime spectroscopy (PALS). PALS is widely recognized as a powerful indirect method to investigate defects in solids. Particularly, it is the only method which gives information on the smallest defects such as single vacancies, small vacancy clusters, voids, etc. The defect structure of an investigated material is reflected in the parameters characterizing a lifetime spectrum. Annihilation lifetimes of positrons trapped at different defects allows, in principle, to determine their types. Moreover, the defect concentration can be estimated from relative intensities of particular components of a lifetime spectrum. Our previous research has shown that unalloyed, bearing and carburizing steels as well as aluminum and titanium alloys, which were subjected to SP, can be successfully investigated by PALS [4, 5]. Also the PALS studies of the surface layer of the stainless steels treated by dry sliding [6] and sandblasting [7] allow to expect that this technique is suitable for similar steels.

The aim of the present work is to determine the influence of the SP parameters on both microstructure (studied by PALS) and mechanical properties (reflected by the surface microhardness) of the material. 


\section{Experimental}

The samples of size $4 \times 15 \times 100 \mathrm{~mm}^{3}$ were made of austenitic stainless and acid resistant steel 1.4541 (EN). The surface of unannealed samples was grinded before they were subjected to pulse SP. The SP work hardening consists of hitting the sample surface with a specified energy and frequency by a ball attached to a slider moving back and forth in a guide. The sample was fixed to a table that was moving at a known speed in the longitudinal and transverse directions. Based on the speed of the table and the frequency of the beating of the beads, the impact density, which is equal to the number of beats per unit area of the sample, was determined. The variable parameters of impulse SP were: impact energy $(E=20 \div 155 \mathrm{~mJ})$, impact density $\left(j=4 \div 44 \mathrm{~mm}^{-2}\right)$ and ball diameter $(D=3.95 \div 12.45 \mathrm{~mm})$. Each of these parameters was varied with other values fixed to: $E=65 \mathrm{~mJ}, j=11 \mathrm{~mm}^{-2}$ and $D=6 \mathrm{~mm}$.

Microhardness was measured by the Vickers method on a LM700AT microhardness tester (LECO). A force of $0.981 \mathrm{~N}$ (HV0.1) or $4.905 \mathrm{~N}$ (HV0.5)was applied to the microindenter.

Scanning electron microscopy (SEM) observations were performed on a TECAN Vega LMU3 microscope in secondary electron mode at high voltage $20 \mathrm{kV}$, magnification $500 \times$ and working distance $30 \mathrm{~mm}$. Oxford energy-dispersive spectrometer (EDS) attached to the microscope was used to check elemental composition of the investigated sample surface.

In PALS measurements, the ${ }^{22} \mathrm{Na}$ source of $60 \mathrm{kBq}$ activity enclosed in Kapton envelope was used. A digital positron lifetime spectrometer, equipped with two detectors with $\mathrm{BaF}_{2}$ scintillators and U1065A high-speed digitizers (Acqiris), was employed. The sampling rate was $8 \mathrm{GS} / \mathrm{s}$. The coincidence unit (Rehberg Electronics) was used as a trigger. Time resolution of the spectrometer was about $203 \mathrm{ps}\left(\mathrm{FWHM}{ }^{22} \mathrm{Na}\right)$. The software was based on the code developed by Prague group [8]. The total count number for each investigated sample was about $10^{7}$. The channel width of 5 ps was chosen. All PALS measurements were carried out in air at room temperature.

The untreated sample and all shot peened ones were subjected to the same studies by all three methods.

\section{Results and discussion}

The chemical composition of the steel under test is presented in Table I.

Only elements with shares exceeding the detection limit of used EDS spectrometer (about $0.1 \mathrm{wt} \%$ ) are included in the table. The contribution of carbon contamination is neglected in the composition calculation. The content of main alloy additives corresponds well to that declared by the manufacturer.

TABLE I

Composition of the surface layer of 1.4541 stainless steel determined by EDS.

\begin{tabular}{c|c|c|c|c|c|c|c}
\hline \hline Element & $\mathrm{Fe}$ & $\mathrm{Cr}$ & $\mathrm{Ni}$ & $\mathrm{Mn}$ & $\mathrm{Si}$ & $\mathrm{P}$ & $\mathrm{S}$ \\
\hline wt\% & $70.4(1)$ & $18.9(1)$ & $8.3(1)$ & $1.7(1)$ & $0.5(1)$ & $0.1(1)$ & $0.1(1)$
\end{tabular}

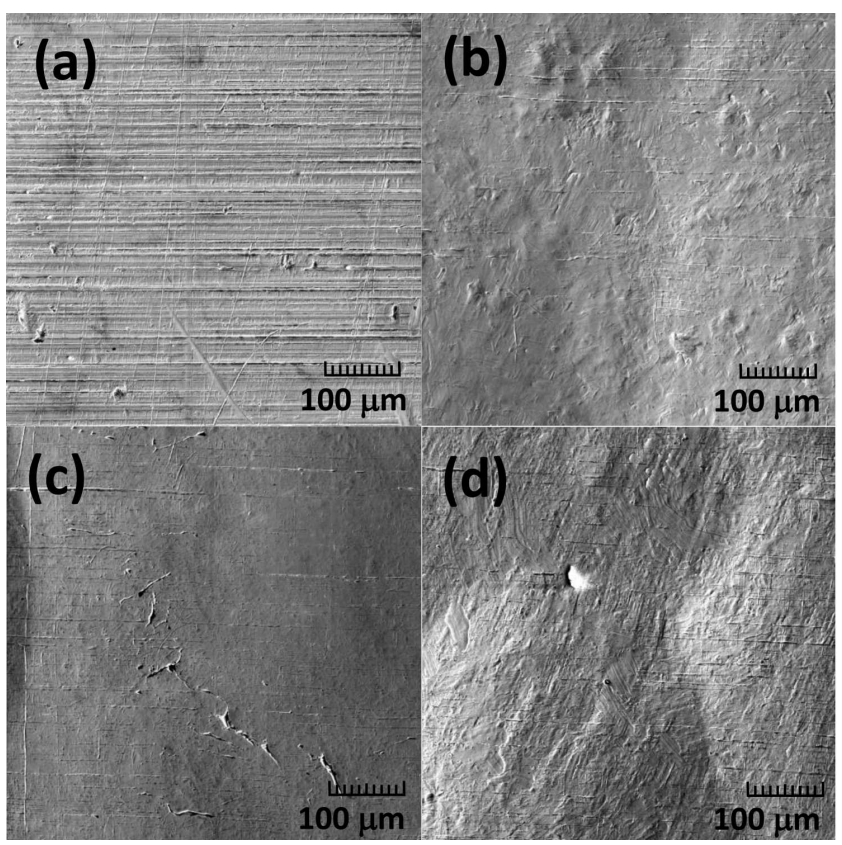

Fig. 1. Low magnification $(500 \times)$ SEM micrographs of the polished un-peened (a), peened at maximum impact energy $E=155 \mathrm{~mJ}$ (b), maximum impact density $j=44 \mathrm{~mm}^{-2}$ (c), and minimum ball diameter $D=3.95 \mathrm{~mm}(\mathrm{~d})$, specimens surface of $1.4541(\mathrm{EN})$ stainless steel.

SEM images for selected samples are shown in Fig. 1. In Fig. 1a the surface morphology of the mechanically grinded sample of commercially available 1.4541 (EN) stainless steel is shown. Abrasion marks and small cracks (black spots) are visible on the surface. After SP the surfaces (Fig. 1b-d) have become corrugated as a result of a moderately strong plastic deformation and simultaneously smoother at small regions - micrometric cracks partially disappear. The SP surface coverage, like for all the investigated shot peened samples discussed in this paper, is more than $100 \%$. The surface of the sample processed with use of the smallest balls seems to have slightly different morphology (with small randomly oriented longitudinal protuberances) than other plastically deformed samples.

Dependences of microhardness on variable impact energy, impact density and ball diameter are plotted in Fig. 2. Except the un-shot peened sample the results of tests are almost the same for $0.981 \mathrm{~N}$ and $4.905 \mathrm{~N}$ loadings. The rapid increase of the surface layer hardness with the increase of both the impact energy $E$ and density $j$ below $c a .20 \mathrm{~mJ}$ and $10 \mathrm{~mm}^{-2}$, respectively, is observed. Next, both dependences achieve a saturation at about $400 \mathrm{HV}$. It suggests that further increase of the energy or density gives no further improvement of mechanical properties of the investigated steel. On the contrary, the effect of a ball diameter decrease has other character. The sample hardness increases almost linearly and does not achieve a saturation in the investigated range. 


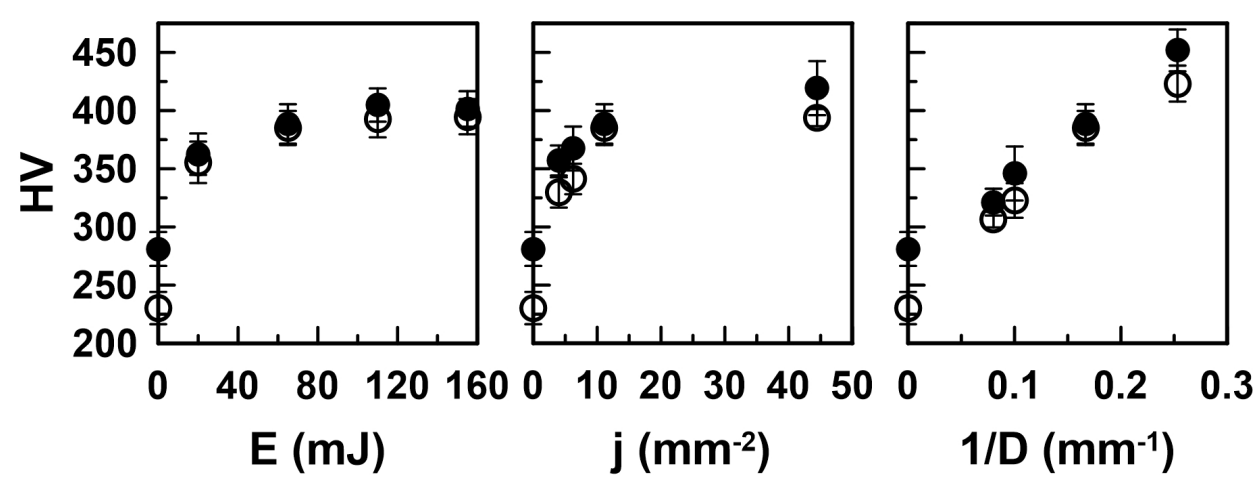

Fig. 2. Microhardness of 1.4541 (EN) stainless steel determined using the force of $0.981 \mathrm{~N}$ (full symbols) and $4.905 \mathrm{~N}$ (open symbols) as a function of impact energy $(E)$, impact density $(j)$ and inverse of ball diameter $(1 / D)$. The SP parameters other than varied in each series were fixed on $E=65 \mathrm{~mJ}, j=11 \mathrm{~mm}^{-2}$ and $D=6 \mathrm{~mm}$.

Indicatively, the significant compressive stresses in shot peened duplex steel occur in the surface layer of thickness of about 150 and $200 \mu \mathrm{m}$ for austenitic and ferritic phases, respectively [2]. The tensile stresses in deeper material layers are considerably smaller and more stretching than in the surface layer. These results are in agreement with PALS results for the stainless steel subjected to dry sliding [6]. However, sandblasting, which seems to interact with the surface in more similar way to shot peening, introduces changes only to $35 \mu \mathrm{m}$. This seems to be result of different energy transferred to the surface by sliding or striking elements.

The penetration depth of positrons that probe a sample has to be estimated to correlate the microhardness and PALS results. From the equation [9]:

$$
P(z)=\exp \left(-\alpha_{+} z\right),
$$

where

$$
\alpha_{+}\left[\mathrm{cm}^{-1}\right]=\left(12.6 Z_{\text {eff }}^{0.17} / E_{\max }^{1.28}\right) \rho\left[\mathrm{g} / \mathrm{cm}^{3}\right],
$$

the mean penetration depth and the information depth of positrons (i.e. $95 \%$ positrons annihilate in a layer of this thickness) for 1.4541 stainless steel $\left(Z_{\text {eff }}=25.43\right.$, $\rho=7.92 \mathrm{~g} / \mathrm{cm}^{3}$ ) were estimated as $27 \mu \mathrm{m}$ and $80 \mu \mathrm{m}$, respectively. More precise calculation of the positron range [10] suggest that it is even somewhat smaller than the estimated values. From the comparison of the above results with the depth of plastically deformed layer it can be stated that positrons allow us to get information only from the upper part of the surface layer.

All obtained spectra were decomposed into three positron annihilation lifetime components with satisfactory $\chi^{2}$ values below 1.25 . The third, longest-lived $\left(\tau_{3} \approx 1.6 \mathrm{~ns}\right)$ component with very small intensity not exceeding of a few tenths of percent was found only due to the very low level of background of random coincidences $(\approx 3$ counts per 5 ps channel). This component can be attributed to pick-off annihilation of ortho-positronium (o-Ps), which forms at the interface between a sample and the Kapton foil enclosing the ${ }^{22} \mathrm{Na}$ source [11, 12]. Because of the small intensity of this component and lack of significant differences between samples, its lifetime was fixed at the average value for all samples and it shall not be taken into account in the further discussion of the results.

In the un-peened 1.4541 stainless steel, two main positron lifetime components: $\left(\tau_{1} \approx 150 \mathrm{ps}, I_{1} \approx 92 \%\right)$ and $\left(\tau_{2} \approx 239 \mathrm{ps}, I_{2} \approx 8 \%\right)$ were revealed. Very close results for positron annihilation parameters in neutron irradiated 1.4301 (EN) stainless steel are reported in [13]. The former component corresponds to annihilation of positrons trapped at monovacancies or divacancies located on the edge dislocations $[14,15]$. The presence of the alloying elements in high-alloyed steel may also influence positron lifetimes. The lifetimes calculated for vacancy-solute complexes for steel alloying elements (e.g. $\mathrm{V}-\mathrm{Cr}$ or $\mathrm{V}-\mathrm{Ni}$ ) are equal to about $152 \div 154 \mathrm{ps}[16,17]$.

The latter component probably origins from the small vacancy clusters that can be estimated as three vacancies [18]. However, the recent positron annihilation lifetime calculations by the method based on the twocomponent density functional theory in $\alpha$-Fe shows that the lifetime of 242 ps corresponds rather to a slightly larger cluster of five vacancies [19]. Similar lifetime was calculated for the cluster of four vacancies in $\mathrm{Ni}$ [20].

The presented result implies almost saturated trapping at dislocations. This, in turn, means that already in the only grinded sample almost all positrons are trapped at vacancies conjugated with dislocations or into small vacancy clusters. In fact, a short-lived ( $c a .60 \mathrm{ps)} \mathrm{compo-}$ nent corresponding to reduced bulk lifetime component (i.e. positrons annihilating from delocalized states in perfect crystal lattice 106 ps $[13,21]$ ) was found to possibly exist only in un-shot peened 1.4541 (EN) SS with the intensity of several percent. However, its short lifetime, low intensity, and large number of other components makes impossible to obtain reliable results with this component taken into account. Therefore, it should be noticed that the presented results are slightly distorted due to neglecting the bulk component.

In the shot peened 1.4541 (EN) stainless steel samples, two main types of defects occur: vacancy-like defects coupled with edge dislocations $\left(\tau_{1} \approx 140 \div 150 \mathrm{ps}\right.$, $I_{1} \approx 55 \div 65 \%$ ) and monovacancies or their small clusters $\left(\tau_{2} \approx 180 \div 200 \mathrm{ps}, I_{2} \approx 35 \div 45 \%\right)$. The bulk 
lifetime is definitely not present in these spectra. The results obtained for a high purity Fe monocrystal show two positron lifetimes: 142 ps - positrons trapped at screw dislocations and $162 \mathrm{ps}$ - positrons trapped at edge dislocations [22]. Probably only the mean lifetime of positrons trapped at these two types of dislocations was observed. Fixing this lifetime to 150 ps allows to estimate, with a reasonable accuracy, the changes of $\tau_{2}$ and $I_{2}$ with the SP parameters (Fig. 3).
The long-lived component changes in the same way with the increase of each SP parameter $(E, j$ and $1 / D)$, namely $\tau_{2}$ decreases and $I_{2}$ increases to reach saturation at $c a .190 \mathrm{ps}$ and $45 \%$. The positron lifetime in a single vacancy in a model alloy of austenitic stainless steels, Fe $14 \mathrm{Cr}-13 \mathrm{Ni}$ was calculated as 183 ps [13]. Thus, single vacancies and divacancies seem to dominate in saturation. Unlike microhardness, there is no different behavior in the $\tau_{2}(1 / D)$ and $I_{2}(1 / D)$ dependences.
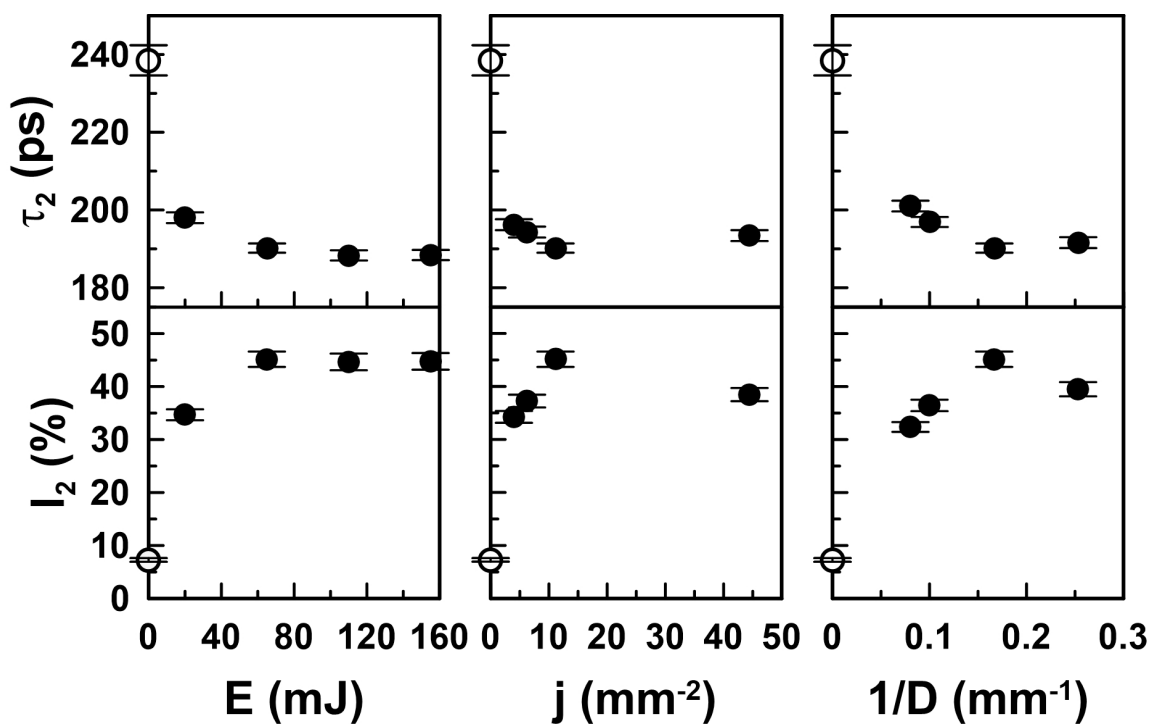

Fig. 3. Lifetime and intensity of the second component in stainless steel 1.4541 (EN) as a function of impact energy $(E)$, impact density $(j)$ and inverse of ball diameter $(1 / D)$. The first component with lifetime of 150 ps was assumed to originate from dislocations [14]. The results for the un-shot peened sample (open symbol) are possibly distorted due to neglecting the low intensity bulk component.
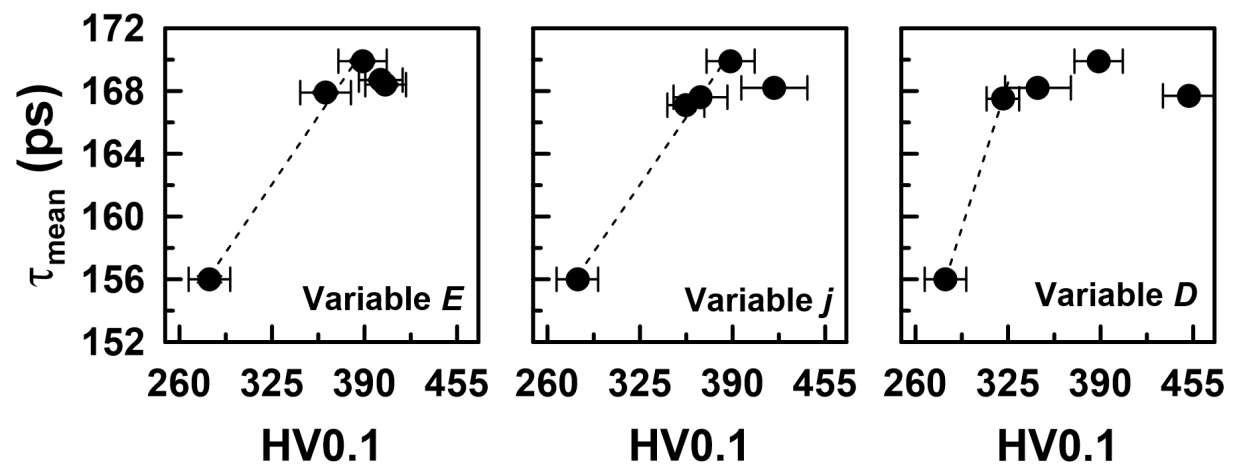

Fig. 4. Mean lifetime of positrons in 1.4541 (EN) stainless steel as a function of microhardness HV0.1. The SP parameters other than varied in each series were fixed on $E=65 \mathrm{~mJ}, j=11 \mathrm{~mm}^{-2}$ and $D=6 \mathrm{~mm}$. The uncertainty of $\tau_{\text {mean }}$ is smaller than the size of symbols $\left(\Delta \tau_{\text {mean }}<1 \mathrm{ps}\right)$.

For the un-shot peened sample the mean lifetime of positron, defined as:

$$
\tau_{\text {mean }}=\sum_{i=1}^{3} I_{i} \tau_{i},
$$

is equal to $156(1)$ ps and for shot peened samples is practically constant and amounts to an average value at approximately 190(9) ps at variable impact energy, 193(13) ps at variable impact density and 185(2) ps with a changing ball diameter. These lifetimes are comparable or even slightly greater to those observed in the stainless steel subjected to dry sliding [6], while sandblasting [7] has effect similar to the one observed in the grinded sample. The data on the mean lifetimes and Vickers hardness numbers measured with changing $E, j$ and $1 / D$ for shot peened samples are compiled in $\tau_{\text {mean }}{ }^{-}$ $\mathrm{HV}$ coordinate system in Fig. 4 . The increase of $\tau_{\text {mean }}$ with improving $\mathrm{HV}$ is clearly visible for the sample series 
as a function of $E$ and $j$. This indicates that sample surface hardness increases as a result of the development of the defect structure in the 1.4541 (EN) stainless steel. It is known that plasticity of material is caused by dislocation mobility. The increasing concentration of vacancylike defects restricts this motion to a large extent. The $\tau_{\text {mean }}(\mathrm{HV})$ relation is quite different for the series SP with balls of various diameter. The saturation of $\tau_{\text {mean }}$ is reached there already at $320 \mathrm{HV} 0.1$ and do not change with further increase of the microhardness. To apply the previous explanation to this case, the formation of vacancies below the information depth of positrons has to be assumed.

\section{Conclusions}

The increase of hardness of the shot peened samples depends on impact energy and density only for initial rapid ramp-up in HV. Further increase of these parameters do not result in hardening of the 1.4541 (EN) steel. On the contrary, the dependence of hardness on the reciprocal of diameter has linear character and does not achieve the saturation, at least in the investigated range. The decrease of a ball diameter allows to obtain the maximum observed hardness of 452(18) HV0.1 and 423(15) HV0.5.

The positron trapping is saturated in almost all samples, most likely due to the strongly non-uniform spatial distribution of dislocations in the samples. It means that positron trapping rate is much greater than positron annihilation rate from the delocalized state in the perfect bulk crystal. Thus, it is impossible to get a quantitative information about trapping rates and concentrations of particular types of defects (e.g. by using the simple trapping model), which are present in the investigated samples. Nevertheless, the presence of the bulk component in the un-shot peened sample indicates that the density of dislocations is near, but still below the upper limit of the PALS sensitivity [23]. Thus, both the dislocations density and vacancies concentration are expected to be below their sensitivity limits, i.e. $5 \times 10^{15} \mathrm{~m}^{-2}$ and $10^{-4}$, respectively. There is definitely no bulk component in the spectra of the shot-peened samples indicating the saturation of positron trapping in defects. The intensity of the vacancy related component is much higher after SP indicating that the ratio of the vacancy concentration and dislocations density increases. However, the plastic deformation caused by SP has to lead to the increase in dislocations density. Therefore, most likely both concentrations are above the upper limit of the PALS sensitivity.

PALS and hardness testing results do not correspond very well due to different depth profiles, characteristic for both methods. This is particularly pronounced in the series of samples shot peened with balls of varying diameters. Such discrepancy can be justified by formation of vacancies that influence hardness below the positron information depth, i.e. $100 \mu \mathrm{m}$.

\section{References}

[1] V. Azar, B. Hashemi, M. Rezaee Yazdi, Surf. Coat. Technol. 204, 3546 (2010).

[2] Q. Feng, C. Jiang, Z. Xu, L. Xie, V. Ji, Surf. Coat. Technol. 226, 140 (2013).

[3] B. Nasiłowska, Z. Bogdanowicz, M. Wojucki, J. Construct. Steel Res. 115, 276 (2015).

[4] R. Zaleski, K. Zaleski, M. Gorgol, M. Wiertel, Appl. Phys. A 120, 551 (2015).

[5] R. Zaleski, M. Gorgol, K. Zaleski, Phys. Proced. 35, 92 (2012).

[6] P. Horodek, J. Dryzek, Mater. Sci. Forum 666, 46 (2011).

[7] P. Horodek, K. Siemek, J. Dryzek, A.G. Kobets, M. Wróbel, Tribol. Lett. 65, 30 (2017).

[8] F. Becvár, J. Cízek, I. Procházka, Acta Phys. Pol. A 113, 1279 (2008).

[9] J. Dryzek, D. Singleton, Nucl. Instrum. Methods Phys. Res. B 252, 197 (2006).

[10] J. Dryzek, K. Siemek, J. Appl. Phys. 114, 224901 (2013).

[11] A. Dupasquier, in: Positrons in Solids, Ed. P. Hautojärvi, Springer-Verlag, Berlin 1979, p. 197.

[12] F. Tuomisto, I. Makkonen, Rev. Mod. Phys. 85, 1583 (2013).

[13] T. Yoshiie, X. Cao, Q. Xu, K. Sato, T.D. Troev, Phys. Status Solidi C 6, 2333 (2009).

[14] J. Čížek, I. Procházka, J. Kočík, E. Keilová, Phys. Status Solidi A 178, 651 (2000).

[15] L. Petrov, T. Troev, N. Nankov, E. Popov, J. Phys. Conf. Series 207, 012037 (2010).

[16] Y. Nagai, K. Takadate, Z. Tang, H. Ohkubo, H. Sunaga, H. Takizawa, M. Hasegawa, Phys. Rev. B 67, 224202 (2003).

[17] J. Kuriplach, O. Melikhova, C. Domain, C.S. Becquart, D. Kulikov, L. Malerba, M. Hou, A. Almazouzi, C.A. Duque, A.L. Morales, Appl. Surf. Sci. 252, 3303 (2006).

[18] M.J. Puska, R.M. Nieminen, J. Phys. F Met. Phys. 13, 333 (1983).

[19] H. Ohkubo, Z. Tang, Y. Nagai, M. Hasegawa, T. Tawara, M. Kiritani, Mater. Sci. Eng. A 350, 95 (2003).

[20] B.L. Shivachev, T. Troev, T. Yoshiie, J. Nucl. Mater. 306, 105 (2002).

[21] W. Zhang, B. Gu, J. Liu, B. Ye, Comp. Mater. Sci. 105, 32 (2015).

[22] Y.-K. Park, J.T. Waber, M. Meshii, C.L. Snead, C.G. Park, Phys. Rev. B 34, 823 (1986).

[23] J. Gubicza, Defect Structure and Properties of Nanomaterials, 2nd and extended ed., Woodhead Publ., Duxford 2017. 